\title{
A Role for Corticotropin-Releasing Factor in Repeated Cold Stress- Induced Anxiety-Like Behavior during Forced Swimming and Elevated Plus-Maze Tests in Mice
}

\author{
Hiroyuki Nishikawa, Taeko Hata,* Eiji Itoh, and Yoshinori Funakami \\ Department of Pharmacology, Faculty of Pharmaceutical Sciences, Kinki University; 3-4-1 Kowakae, Higashi-Osaka \\ 577-8502, Japan. Received November 18, 2003; accepted December 24, 2003; published online January 5, 2004
}

\begin{abstract}
SART (specific alternation of rhythm in temperature) stress is known to cause anxiety-like behavior in mice/rats in several anxiety-related behavioral tests. In the present study, we investigated possible roles for corticotropin-releasing factor (CRF) and glucocorticoids in SART stress-induced anxiety-like behavior in two different anxiety-related behavioral tests. In the forced swimming test, CRF, administered intracerebroventricular (i.c.v.) at $0.5-2 \mathrm{pmol} / \mathrm{mouse}$, dose-dependently reduced immobility time in unstressed and SART-stressed mice. $\alpha$-Helical CRF, a specific CRF receptor antagonist, administered i.c.v. at $0.1-1 \mathrm{nmol} / \mathrm{mouse}$, dose-dependently increased immobility time in SART-stressed mice, but not in unstressed mice. In the elevated plus-maze test, CRF at $10-20 \mathrm{pmol} / \mathrm{mouse}$ significantly decreased the time spent in open arms in unstressed mice. CRF at a high dose tended to decrease this time in SART-stressed mice, but this decrease was not statistically significant. $\alpha$-Helical CRF failed to modify the time in unstressed mice. In contrast, $\alpha$-helical CRF at 0.38 and $0.75 \mathrm{nmol} / \mathrm{mouse}$ increased the time in SART-stressed mice. Both immobility time in the forced swimming test and time spent in open arms in the elevated plus-maze test in unstressed and SART-stressed mice were unaffected by adrenalectomy. These results suggest that CRF plays an important role in anxiety-like behavior caused by SART stress.
\end{abstract}

Key words anxiety; repeatedly cold stress; corticotropin-releasing factor (CRF); specific alternation of rhythm in temperature (SART) stress; elevated plus-maze test; forced swimming test

Anxiety is one of the most prominent psychiatric disorder related to a common stress. The success of pharmacological treatments for this disorder has been dampened by various factors, including resistance to treatment and adverse effects of the drugs used. Although the themes of stress and anxiety have long been a topic of investigation, current anxiolytic drugs are only based on pharmacological interactions with classic neurotransmitters. Accordingly, we investigated possible alternative mechanisms that might lead to development of new classes of anxiolytic drugs using SART-stressed animals.

Corticotropin-releasing factor (CRF), a hypothalamic peptide consisting of 41 amino acids, is a key mediator of mammalian endocrine, behavioral, autonomic, and immune responses to stress. ${ }^{1,2)}$ Within the hypothalamic-pituitaryadrenal (HPA) axis, CRF is the principal regulator of pituitary adrenocorticotropic hormone (ACTH) and adrenal glucocorticoid secretion in responses to stressful stimuli. Basic research studies indicate that elevated central CRF levels are involved in the etiology of stress-related psychiatric, physiological and behavioral disorders. ${ }^{3)}$ Central administration of CRF exerts potent anxiogenic effects in experimental animals. ${ }^{4)}$ Clinical studies also demonstrate that $\mathrm{CRF}$ is implicated in anxiety. ${ }^{5,6)}$

We have investigated the effects of stress on physiological conditions and on emotions using SART (specific alternation of rhythm in temperature) stress, ${ }^{7}$ which is known as a model of autonomic imbalance. ${ }^{8)}$ The stressful situation is created by repeated and sudden changes in environmental temperature from room temperature to cold temperature, an event that may be encountered by humans in daily life such as in early spring or autumn, or when leaving an air-conditioned room in summer or a heated room in winter to go outdoors. Animals exposed to SART stress show adverse biological events ${ }^{9-11)}$ and physiological abnormalities. ${ }^{7,12,13)}$ Fur- thermore, a series of our behavioral studies using SARTstressed animals demonstrated that environmental stress induces anxiety-like behavior. ${ }^{14-16)}$ The anxiety-like behaviors caused by SART stress are normalized by anxiolytic agents such as diazepam, alprazolam, benzodiazepine-receptor agonists, and buspirone, a selective $5-\mathrm{HT}_{1 \mathrm{~A}}$ serotonin-receptor agonist. ${ }^{14-16)}$ This evidence suggests that SART-stressed animals may be in a state of anxiety.

As SART stress causes anxiety-like behavior in mice and rats in several tests, we turned our attention to the possibility that CRF may be involved in the stress and anxiety of SARTstressed mice. In the present study, we determined whether inhibition of CRF receptor function using $\alpha$-helical CRF as a specific CRF receptor antagonist could normalize anxietylike behavior caused by SART stress using forced swimming (FS) test and elevated plus-maze (EPM) test.

The FS test is a well-known screening model for antidepressants developed by Porsolt et al. ${ }^{17,18)}$ At first, they suggested that the characteristic immobility observed in the FS test reflects a state of "despair" in the rodents. However, some studies employing the FS test have questioned its specificity for predicting antidepressant activity, since not only antidepressants but also many other drugs have been equally effective in the test. ${ }^{19,20)}$ Moreover, other studies have revealed that anxiolytics enhance the prolongation of immobility time $^{21,22)}$ while anxiogenic agents reduced the time ${ }^{22)}$ in rodents. These evidences suggest that the behavior of rodents in the FS test might be related to fear and/or anxiety.

The EPM test is widely accepted as a method for deteremining the anxiety state in rodents. ${ }^{23)}$ Mice and rats, allowed to freely explore the EPM apparatus, spent less time on the open arms than on the closed arms of the apparatus. Anxiolytics increase the time spent on the open arms but not the number of entries into the open and closed arms. 
Furthermore, we evaluated the role of the glucocorticoids on SART stress-induced anxiety-like behavior using adrenalectomy (ADX).

Here we report that suppression of CRF receptor function normalizes anxiety-like behavior in mice caused by SART stress.

\section{MATERIALS AND METHODS}

Animals Male ddY mice (Japan SLC, Hamamatsu), weighing $23-28 \mathrm{~g}$ at the start of the study, were used in accordance with ethical procedures approved for the care and use of laboratory animals by the Japanese Pharmacological Society. They were housed in groups of $8-10$ in plastic cages $(21.6 \mathrm{~cm} \times 31.6 \mathrm{~cm} \times 13.0 \mathrm{~cm})$ and allowed standard laboratory diet and tap water ad libitum. All mice were maintained under standard laboratory conditions $\left(24 \pm 1^{\circ} \mathrm{C}, 12-\mathrm{h}\right.$ light/dark cycle with light onset at 07:00 h).

Peptides CRF and $\alpha$-helical CRF (Sigma Chemical Co., St. Louis, Missouri, U.S.A.) were used.

For intracerebroventricular (i.c.v.) administration, these peptides were dissolved in distilled water adjusted to $\mathrm{pH} 6.7$ with $1 \mathrm{~N} \mathrm{HCl}$ and injected in a volume of $5 \mu \mathrm{l}$ per mouse according to the direct injection method of Brittain and Handley ${ }^{24)} 5 \mathrm{~min}$ before the test.

Exposure of Animals to SART Stress Mice were stressed essentially according to a previously established protocol. ${ }^{25)}$ In brief, plastic cages $(21.6 \mathrm{~cm} \times 31.6 \mathrm{~cm} \times 13.0 \mathrm{~cm})$ for stress exposure were prepared in a room maintained at $24{ }^{\circ} \mathrm{C}$ and others in a room maintained at $4{ }^{\circ} \mathrm{C}$. Mice in groups of $8-10$ were alternately transferred at $1-\mathrm{h}$ intervals to cages placed in the two rooms between 09:00 and 16:00 $\mathrm{h}$ and housed in cages in the latter room $\left(4^{\circ} \mathrm{C}\right)$ between 16:00 and 09:00 h overnight. These procedures were repeated for $6-8 \mathrm{~d}$ and thereafter terminated at $11: 00 \mathrm{~h}$ on the final day of stress. The stressed mice were used a $0.5 \mathrm{~h}$ after being taken out of the cold room $\left(4^{\circ} \mathrm{C}\right)$ or later.

Forced Swimming Test The FS test was conducted according to the method of Porsolt et al. ${ }^{17,18)}$ Briefly previously mice were forced to swim in a glass cylinder $(8 \mathrm{~cm}$ in diameter and $20 \mathrm{~cm}$ in height) containing fresh water (at $22 \pm 1^{\circ} \mathrm{C}$ ) added to a height of $8 \mathrm{~cm}$. Initially, the mice vigorously swam around. A few minutes later, their activity began to subside and eventually they ceased to move and floated on the water in an upright position, making only small movements to keep their heads above the water. The length of time that each animal remained immobile was measured over a 5 min period. When two or more mice were tested simultaneously, the cylinders were hidden from each other using opaque panels on three sides.

Elevated Plus-Maze Test The EPM test ${ }^{23)}$ was conducted as previously described with some modifications. The apparatus consisted of two open arms $(5 \times 30 \mathrm{~cm})$ and two closed arms $(5 \times 30 \times 15 \mathrm{~cm})$ radiating from a central platform $(5 \times 5 \mathrm{~cm})$ to form a plus-sign figure. The apparatus was situated $40 \mathrm{~cm}$ above the floor. The open-arm edges were $0.5 \mathrm{~cm}$ in height to keep the mice from falling, and the closed-arm edges were $15 \mathrm{~cm}$ in height. The mice were individually examined in 5-min sessions in this apparatus. Each mouse was placed in the central platform facing one open arm. The number of entries into open and closed arms and the time spent on the respective arms were recorded for a 5 min period.

Adrenalectomy (ADX) ADX was performed in mice essentially according to a previously established protocol. ${ }^{26)}$ In brief, both adrenal glands were removed under anesthesia (using pentobarbital at $40 \mathrm{mg} / \mathrm{kg}$ ), through incisions in the back, just below the breast ribs. Afterwards, the skin was sutured. Sham operated mice were subjected to the same surgical procedure as ADX mice, but the adrenals were left intact. After surgery, including sham operation, tap water available to the mice was replaced by a $0.9 \% \mathrm{NaCl}$ solution. Mice were used for experiments $6 \mathrm{~d}$ after surgery.

Statistical Analysis Data are presented as mean \pm S.E. of the mean, and statistical significance was analyzed by the Student's $t$-test or analysis of variance (ANOVA) followed by Tukey's multiple-comparison test. $p$-Values less than 0.05 were considered significant.

\section{RESULTS}

Effects of CRF and $\alpha$-Helical CRF on Immobility Time of Unstressed and SART-Stressed Mice in the FS Test Immobility time following i.c.v. administration of CRF or $\alpha$ helical CRF are shown in Figs. 1 and 2. CRF doses of $0.5,1$ and $2 \mathrm{pmol} / \mathrm{mouse}$ dose-dependently reduced immobility time in unstressed (Fig. 1a) and SART-stressed mice (Fig. 1b). $\alpha$-Helical CRF doses of $0.1,0.3$ and $1 \mathrm{nmol} / \mathrm{mouse}$, dose-dependently increased the immobility time in SARTstressed mice (Fig. 2b), but had no effect on unstressed mice (Fig. 2a).
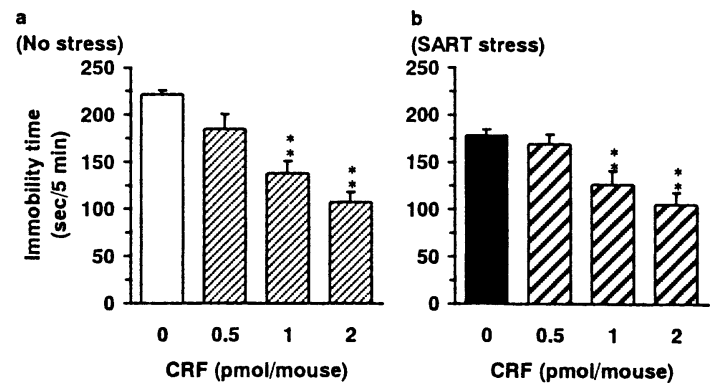

Fig. 1. Effect of CRF on Immobility Time of Unstressed and SARTStressed Mice in the Forced Swimming Test

$\mathrm{CRF}$ or vehicle was administered i.c.v. $20 \mathrm{~min}$ before the test. Data show the mean with S.E. of the mean. $n=11$ (vehicle; dose $=0$ ) or $6-8$ (treated with CRF). $* * p<0.01$ vs. the vehicle-treated group (Tukey's test).
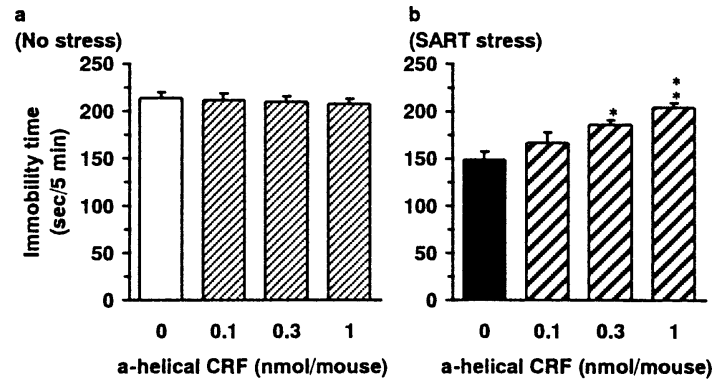

Fig. 2. Effect of $\alpha$-Helical CRF, a Specific CRF Antagonist, on Immobility Time of Unstressed and SART-Stressed Mice in the Forced Swimming Test

$\alpha$-Helical CRF or vehicle was administered i.c.v. 20 min before the test. Data show the mean with S.E. of the mean. $n=8 . * p<0.05$ and $* * p<0.01 v s$. the vehicle-treated $($ dose $=0)$ group (Tukey's test). 

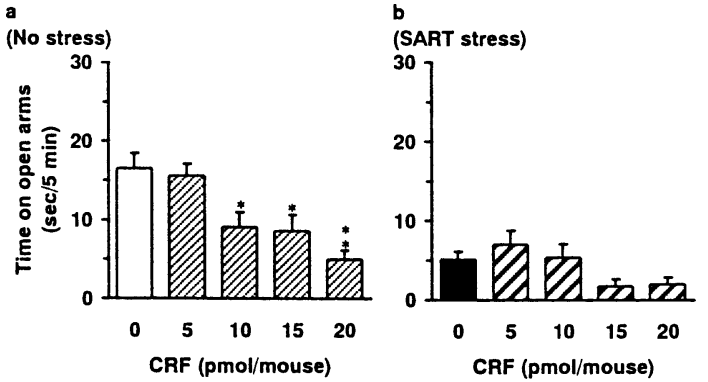

Fig. 3. Effect of CRF on SART Stress-Induced Anxiety-Like Behavior in the Elevated Plus-Maze Test

CRF was administered i.c.v. $20 \mathrm{~min}$ before the test. Data show the mean with S.E. of the mean. $n=6-8 . * p<0.05$ and $* * p<0.01 v s$. the vehicle-treated (dose $=0)$ group (Tukey's test).
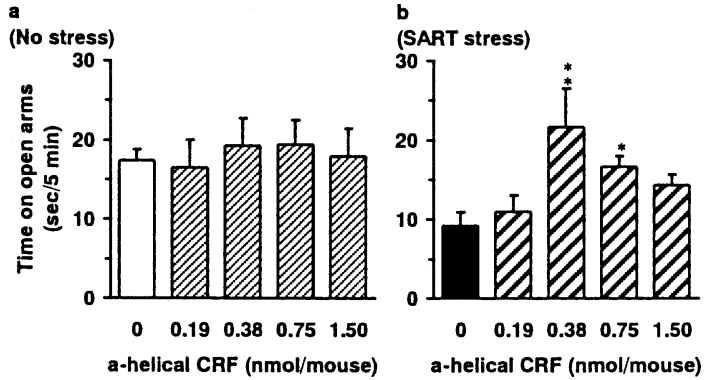

Fig. 4. Effect of $\alpha$-Helical CRF on SART Stress-Induced Anxiety-Like Behavior in the Elevated Plus-Maze Test

$\alpha$-Helical CRF was administered i.c.v. $20 \mathrm{~min}$ before the test. Data show the mean with S.E. of the mean. $n=14$ or 15 (vehicle; dose $=0$ ) or $5-10$ (treated with CRF). $* p<0.05$ and $* * p<0.01$ vs. the vehicle-treated group (Tukey's test).

Effects of CRF and $\alpha$-Helical CRF on SART Stress-Induced Anxiety-Like Behavior in the EPM Test The time spent on open arms in the EPM test following the i.c.v. administration of CRF is shown in Fig. 3. CRF doses between $10-20 \mathrm{pmol} / \mathrm{mouse}$ significantly decreased the time spent on open arms by unstressed mice (Fig. 3a). In the SART-stressed mice, however, only CRF at 15 and $20 \mathrm{pmol} / \mathrm{mouse}$ decreased the time, and this decrease was not statistically significant (Fig. 3b). As shown in Fig. 4a, $\alpha$-helical CRF failed to modify the time spent on open arms in unstressed mice. In contrast, $\alpha$-helical CRF at 0.38 and $0.75 \mathrm{nmol} / \mathrm{mouse}$ increased it in SART-stressed mice. This response was biphasic, however, since higher doses (e.g., $1.5 \mathrm{nmol} /$ mouse) failed to elicit an increase (Fig. 4b).

Influence of ADX on Anxiety-Like Behavior Observed in SART-Stressed Mice in FS and EPM Tests As shown in Table 1, immobility time of unstressed mice in the FS test was unaffected by ADX. Also the shortened immobility time in SART-stressed mice was not altered by ADX. Similarly, the time spent on open arms in the EPM test was unaffected by ADX in unstressed and also in SART-stressed mice, though non-significant increases were observed in both groups of mice (Table 2).

\section{DISCUSSION}

The present study demonstrates that SART stress-induced anxiety-like behavior observed in two different anxiety-related behavior tests, FS and EPM tests, is normalized by $\alpha$ -
Table 1. Influence of Adrenalectomy (ADX) on the Immobility Time of Unstressed and SART-Stressed Mice in the Forced Swimming Test

\begin{tabular}{lcc}
\hline \hline & \multicolumn{2}{c}{ Duration of immobility (s/5 min) } \\
\cline { 2 - 3 } & Sham operated group & ADX \\
\hline No stress & $208.7 \pm 3.2(11)$ & $206.6 \pm 3.4(13)$ \\
SART stress & $172.0 \pm 5.6(10)$ & $173.5 \pm 4.1(14)$ \\
\hline $\begin{array}{l}\text { Data show the mean with S.E. of the mean. Numbers in parentheses show numbers } \\
\text { of mice. }\end{array}$
\end{tabular}

Table 2. Influence of ADX on SART Stress-Induced Anxiety-Like Behavior in the Elevated Plus-Maze Test

\begin{tabular}{lcr}
\hline \hline & \multicolumn{2}{c}{ Time on open arms (s/5 min) } \\
\cline { 2 - 3 } & Sham operated group & ADX \\
\hline No stress & $10.4 \pm 1.8(12)$ & $14.1 \pm 1.4(13)$ \\
SART stress & $4.0 \pm 1.4(12)$ & $7.2 \pm 1.0(13)$ \\
\hline
\end{tabular}

Data show the mean with S.E. of the mean. Numbers in parentheses show numbers of mice.

helical CRF, a specific CRF receptor antagonist, but not by ADX. We conclude that CRF plays an important role in anxiety-like behavior caused by SART stress.

SART-stressed animals exhibit abnormal behavior in openfield, ${ }^{27)}$ step-down ${ }^{28)}$ and FS tests. ${ }^{14,15)}$ The open-field behavior observed in SART-stressed rats, increases in locomotor activity, rearing and defecation and decrease in grooming, was partially inhibited by anxiolytics such as diazepam and alprazolam. ${ }^{27)}$ The shortened immobility time in the FS test was also improved by these drugs. ${ }^{14)}$ Moreover, the physiological abnormalities of SART-stressed animals, such as prolongation of the QRS interval in the electrocardiogram $(\mathrm{ECG})^{13)}$ and electroencephalographic (EEG) alterations, ${ }^{12}$ were normalized by diazepam and alprazolam. These findings suggest that SART-stressed animals are very likely in a state of anxiety.

CRF has been identified as a neuropeptide that plays a central role in the coordination of neuroendocrine, autonomic, and behavioral responses to stress-induced release of ACTH. ${ }^{29)}$ Two subtypes of the CRF receptor, $\mathrm{CRF}_{1}$ and $\mathrm{CRF}_{2}$, each having a distinct anatomical localization and pharmacology have been identified. ${ }^{30)}$ During the past few years, many studies have been conducted to discern the roles of $\mathrm{CRF}_{1}$ and $\mathrm{CRF}_{2}$ in stress-related physiological and behavioral processes to gain insight into anxiety. Various strategies have been employed, including approaches from pharmacology and from molecular biology. Several lines of evidence indicate the participation of $\mathrm{CRF}_{1}$ in mediating the effects of CRF. First, $\mathrm{CRF}_{1}$, but not $\mathrm{CRF}_{2}$, binds $\mathrm{CRF}$ with high affinity. ${ }^{31)}$ Second, $\mathrm{CRF}_{1}$-deficient mice show reduced anxiety-related behavior. ${ }^{32)}$ Third, the selective $\mathrm{CRF}_{1}$ receptor antagonists inhibit the anxiogenic action of $\mathrm{CRF}^{33)}$ Although there is robust evidence that $\mathrm{CRF}_{1}$ is intimately involved in anxiety-related behavior, a role for $\mathrm{CRF}_{2}$ cannot be excluded. In $\mathrm{CRF}_{2}$-deficient mice, anxiety-related behavior was observed to increase. ${ }^{34)}$ But this evidence does not provide a clear answer to the question about the role of $\mathrm{CRF}_{2}$ in anxiety. These studies have provided insight into the complex roles of $\mathrm{CRF}_{1}$ and $\mathrm{CRF}_{2}$ in the regulation of emotional behavior, HPA axis 
activity and autonomic function. Some roles of $\mathrm{CRF}_{1}$ and $\mathrm{CRF}_{2}$ seem to be clear, whereas others still need to be resolved. Collectively, CRF is a key mediator of psychological reaction, emotion and stress.

Behavior in the EPM is a model for anxiety in rodents and may serve as a new basis for developing anxiolytic agents and investigating psychological and neurochemical factors in anxiety. Mice and rats, allowed to freely explore the EPM apparatus, spent less time exploring the open arms than the closed arms of the apparatus. SART stress significantly reduced the time spent on the open arms but not the numbers of entries into the open and closed arms. ${ }^{16)}$ Diazepam, alprazolam and buspirone improved the abnormal and anxiety-like behavior in SART-stressed mice in the EPM test without any influence on unstressed mice. ${ }^{16)}$ In this study, CRF produced a decrease in the time spent on the open arms in unstressed and SART-stressed mice. $\alpha$-Helical CRF produced an increase in the time in SART-stressed mice, but had no effect on unstressed mice. It is of note that the effect of the high dose of $\alpha$-helical CRF was less than that of the low dose of $\alpha$-helical CRF. The experiments in other laboratory indicated that the low dose of $\alpha$-helical CRF had no effect on the EPM test in unstressed mice, and that higher doses of the $\alpha$-helical CRF did not produce an anti-stress action in stressed mice. In addition, the higher doses produced even a CRF-like action in unstressed mice. ${ }^{35-37)}$ It is clear from the present study that CRF plays an important role in the anxiety-like behavior induced by SART stress.

Porsolt et al. ${ }^{17,18)}$ suggested that the characteristic immobility observed in the FS test reflects a state of "despair" in the rodents and showed that this immobility is reduced by a variety of agents that are therapeutically effective in depression. On the other hand, some studies employing the FS test have questioned its specificity for predicting antidepressant activity, since not only antidepressants but also many other drugs have been equally effective in this test. ${ }^{19,20)}$ Pharmacological studies have revealed that anxiolytics such as diazepam enhance the prolongation of immobility time in mice $^{21,22)}$ while anxiogenic agents such as $\beta$-carboline reduce the time. ${ }^{22)}$ These studies suggest that the behavior of rodents in the FS test might be related to fear and/or anxiety. SARTstressed mice exhibited a shortened immobility time in the FS test. ${ }^{14,15)}$ The shortened immobility time was normalized by anxiolytic agents such as diazepam and alprazolam, benzodiazepine receptor agonists, ${ }^{14)}$ and suggested to be related to anxiety. In this study, $\alpha$-helical CRF increased the time in SART-stressed mice, with no effect on unstressed mice. This finding in the FS test indicates also that CRF may be the key mediator of the anxiety-like behavior that is induced by SART stress.

The regulation of glucocorticoid synthesis and release is tightly controlled by the HPA axis. ${ }^{38)}$ Stress and other stimuli induce synthesis and release of CRF from the hypothalamus. CRF leads to an increase in synthesis and secretion of ACTH from the anterior pituitary, thereby stimulating glucocorticoid production and release in the adrenal cortex. Cortisol is the principal circulating glucocorticoid in humans, while corticosterone has this function in rodents. ${ }^{39)}$ Basic research has demonstrated the relationship of corticosteroids to behavioral responses in animal models of anxiety. Administration of corticosterones produces anxiogenic effects. ${ }^{40)}$ Clinical stud- ies have shown that dysregulation and dysfunction of corticosteroid receptors have been implicated in the pathogenesis of depression and anxiety. ${ }^{41,42)}$ These data suggest that glucocorticoid plays an important role in stress-related psychiatric disorders such as anxiety.

We evaluated a role for glucocorticoid in SART stress-induced anxiety-like behavior using ADX, because CRF is an important molecule in the regulation of glucocorticoid synthesis in the HPA axis. Anxiety-like behavior of SARTstressed mice in two different anxiety-related behavior tests was unaffected by ADX. Thus, glucocorticoids are not involved in the anxiety-like behavior caused by SART stress in these tests.

In conclusion, the decreased sensitivity to exogenous CRF and the enhanced responsive to a CRF antagonist observed in the SART-stressed mice are caused by a difference in activity of endogenous CRF pathways which could be reflected in either amount or affinity of CRF receptors. This indicates that CRF receptor and/or CRF pathways are highly involved in anxiety-like behavior induced by SART stress.

\section{REFERENCES}

1) Vale W., Spiess J., Rivier C., Rivier J., Science, 213, 1394-1397 (1981).

2) Owens M. J., Nemeroff C. B., Pharmacol. Rev., 43, 425-473 (1991).

3) Linthorst A. C., Flachskamm C., Hopkins S. J., Hoadley M. E., Labeur M. S., Holsboer F., Reul J. M., J. Neurosci., 17, $4448-4460$ (1997).

4) Sutton R. E., Koob G. F., Le Moal M., Rivier J., Vale W., Nature (London), 297, 331-333 (1982).

5) Nemeroff C. B., Widerlov E., Bissette G., Walleus H., Karlsson I., Eklund K., Kilts C. D., Loosen P. T., Vale W., Science, 226, 1342-1344 (1984).

6) Holsboer F., Von Bardeleben U., Gerken A., Stalla G. K., Muller O. A., N. Engl. J. Med., 311, 1127 (1984).

7) Kita T., Hata T., Yoneda R., Okage T., Nippon Yakurigaku Zasshi, 71, 195-210 (1975).

8) Kita T., Hata T., Itoh E., Namimatsu A., Jpn. J. Psychosom. Med., 23 , 61-68 (1983).

9) Uchida S., Takeyasu K., Noguchi Y., Yoshida H., Hata T., Kita T., Life Sci., 22, 2197-2203 (1978).

10) Kawabata A., Hata T., Br. J. Pharmacol., 119, 346-350 (1996).

11) Kawabata A., Hata T., Thromb. Res., 72, 321-331 (1993).

12) Hata T., Nishimura Y., Kita T., Kawabata A., Itoh E., Jpn. J. Pharmacol., 45, 365-372 (1987).

13) Hata T., Kita T., Itoh E., Namimatsu A., Nippon Yakurigaku Zasshi, 79, 487-492 (1982).

14) Hata T., Itoh E., Nishikawa H., Pharmacol. Biochem. Behav., 51, 849-853 (1995)

15) Hata T., Nishikawa H., Itoh E., Watanabe A., Jpn. J. Pharmacol., 79, 243-249 (1999)

16) Hata T., Nishikawa H., Itoh E., Funakami Y., Jpn. J. Pharmacol., 85, 189-196 (2001).

17) Porsolt R. D., Bertin A., Jalfre M., Arch. Int. Pharmacodyn. Ther., 229, 327-336 (1977).

18) Porsolt R. D., Le Pichon M., Jalfre M., Nature (London), 266, 730732 (1977).

19) Betin C., DeFeudis F. V., Blavet N., Clostre F., Physiol. Behav., 28, 307-311 (1982)

20) Devoize J. L., Rigal F., Eschalier A., Trolese J. F., Renoux M., Psychopharmacology (Berl), 84, 71-75 (1984).

21) Nagatani T., Sugihara T., Kodaira R., Eur. J. Pharmacol., 97, 271275 (1984).

22) Nagatani T., Yamamoto T., Sugihara T., Ueki S., Eur. J. Pharmacol., 142, 17-22 (1987).

23) Lister R. G., Psychopharmacology (Berl), 92, 180-185 (1987).

24) Brittain R. T., Handley S. L., J. Physiol., 192, 805-813 (1967).

25) Hata T., Kita T., Itoh E., Harada N., Jpn. J. Psychosom. Med., 24, $256-266$ (1984). 
26) Urani A., Roman F. J., Phan V. L., Su T. P., Maurice T., J. Pharmacol. Exp. Ther, 298, 1269-1279 (2001).

27) Hata T., Nishimura Y., Kita T., Itoh E., Kawabata A., Jpn. J. Pharmacol., 48, 479-490 (1988).

28) Nishimura Y., Hata T., Kawabata A., Itoh E., Kita T., Jpn. J. Pharmacol., 49, 111-117 (1989).

29) Reul J. M., Holsboer F., Curr. Opin. Pharmacol., 2, 23-33 (2002).

30) Chalmers D. T., Lovenberg T. W., Grigoriadis D. E., Behan D. P., De Souza E. B., Trends Pharmacol. Sci., 17, 166-172 (1996).

31) Reul J. M., Holsboer F., Curr. Opin. Pharmacol., 2, 23-33 (2002).

32) Timpl P., Spanagel R., Sillaber I., Kresse A., Reul J. M., Stalla G. K., Blanquet V., Steckler T., Holsboer F., Wurst W., Nat. Genet., 19, 162 166 (1998).

33) Okuyama S., Chaki S., Kawashima N., Suzuki Y., Ogawa S., Nakazato A., Kumagai T., Okubo T., Tomisawa K., J. Pharmacol. Exp. Ther. 289, 926-935 (1999).

34) Bale T. L., Contarino A., Smith G. W., Chan R., Gold L. H., Saw- chenko P. E., Koob G. F., Vale W. W., Lee K. F., Nat. Genet., 24, 410414 (2000).

35) Baldwin H. A., Rassnick S., Rivier J., Koob G. F., Britton K. T., Psychopharmacology, 103, 227-232 (1991).

36) Heinrichs S. C., Merlo P. E., Miczek K. A., Britton K. T., Koob G. F., Brain Res., 581, 190-197 (1992).

37) Menzaghi F., Howard R. L., Heinrichs S. C., Vale W., Rivier J., Koob G. F., J. Pharm. Exp. Ther, 267, 564-572 (1994).

38) Palkovits M., Prog. Brain Res., 72, 47-55 (1987).

39) Gass P., Reichardt H. M., Strekalova T., Henn F., Tronche F., Physiol. Behav., 73, 811-825 (2001).

40) Smythe J. W., Murphy D., Timothy C., Costall B., Pharmacol. Biochem. Behav., 56, 507-513 (1997).

41) De Kloet E. R., Vreugdenhil E., Oitzl M. S., Joels M., Endocr. Rev., 19, 269-301 (1998).

42) Brown E. S., Rush A. J., McEwen B. S., Neuropsychopharmacology, 21, 474- 484 (1999). 